\title{
ESTUDO DA ADSORÇÃO DE CHUMBO UTILIZANDO COMO ADSORVENTE BAGAÇO DE CANA-DE-AÇÚCAR ATIVADO
}

\author{
J. E. M. PAZ ${ }^{1}$, A. I. C. GARNICA ${ }^{1 *}$, F. D. S. CURBELO ${ }^{1}$ \\ Universidade Federal da Paraíba ${ }^{1}$ \\ alfredocurbelo@yahoo.com*
}

Submetido 25/07/2018 - Aceito 19/12/2018

DOI: $10.15628 /$ holos.2018.7544

\section{RESUMO}

Neste estudo, avaliou-se a capacidade do bagaço de cana-de-açúcar, modificado quimicamente, para a retenção de íons metálicos de chumbo. A modificação refere-se a um tratamento com ácido sulfúrico $(1,0$ $\mathrm{molL}^{-1}$ ) que leva à reação de grupos carboxilato com a celulose da biomassa. As soluções sintéticas de efluente foram preparadas a partir do nitrato de chumbo $\left(\mathrm{Pb}\left(\mathrm{NO}_{3}\right)_{2}\right)$, obtendo concentrações de 2 a 80 $\mathrm{mgL}^{-1}$ do metal. Os ensaios de adsorção foram desenvolvidos pelo método do banho finito. Estes experimentos foram realizados nas temperaturas de 30,45 e $60{ }^{\circ} \mathrm{C}$ com tempo de equilíbrio de 24 horas. $A$ solução remanescente foi analisada por técnica de espectrofotometria para determinação da concentração do metal. Os resultados experimentais mostraram a maior capacidade de adsorção foi alcançada para a temperatura de $30{ }^{\circ} \mathrm{C}$, sendo de aproximadamente $4,8 \mathrm{mg}$ de metal adsorvido por grama de adsorvente. 0 modelo de Langmuir foi o que que melhor descreveu o processo de adsorção, sendo o de Freundlich não significativo estatisticamente. Da analises dos parâmetros termodinâmicos foi possível constatar o caráter exotérmico do processo estudado, o que condiz com os melhores resultados obtidos para a adsorção do chumbo que foram apresentados para a menor temperatura estudada $\left(30{ }^{\circ} \mathrm{C}\right)$. O sistema também indicou ser um processo espontâneo, através dos valores negativos obtidos para a energia libre de Gibbs e ser configurado como uma adsorção física dado o valor obtido para a entalpia do processo, aproximadamente $-7,55 \mathrm{KJ} / \mathrm{Kmol}$.

PALAVRAS-CHAVE: adsorção, bagaço de cana-de-açúcar, chumbo, modelo de Langmuir

\section{STUDY OF PLUMBUM ADSORPTION USING AS ADSORVENT ACTIVATED SUGAR CANE BAGASSE}

\begin{abstract}
In this study, the capacity of chemically modified sugarcane bagasse, for the retention of metallic ions of plumbum was evaluated. The modification was realized by sulfuric acid $\left(1.0 \mathrm{molL}^{-1}\right)$, and carboxylate groups react with biomass cellulose. The synthetic effluent solutions were prepared from $\mathrm{Pb}\left(\mathrm{NO}_{3}\right)_{2}$, obtaining concentrations of 2 to $80 \mathrm{mgL}^{-1}$ of the metal. The adsorption tests were developed by finite bath method. These experiments were carried out at temperatures of 30,45 and $60{ }^{\circ} \mathrm{C}$ with equilibrium time of 24 hours. The concentration of the metal in solution was analyzed by spectrophotometric technique. The experimental results showed the highest adsorption capacity was
\end{abstract}

reached at $30{ }^{\circ} \mathrm{C}$, with approximately $4.8 \mathrm{mg}$ of adsorbed metal per gram of adsorbent. The adsorption process was better described by Langmuir model and Freundlich's model was not significant statistically. By thermodynamic parameters, it was possible to verify the exothermic character of the studied process which it is agree with the best results obtained for the adsorption at the lowest temperature studied $\left(30{ }^{\circ} \mathrm{C}\right)$. The system also indicated to be a spontaneous process, through the negative values obtained for the free energy of Gibbs and to be configured as a physical adsorption, according the value obtained for the enthalpy of the process, approximately, $-7.55 \mathrm{KJ} / \mathrm{Kmol}$.

KEYWORDS: adsorption, sugarcane bagasse, Plumbum, Langmuir model 


\section{INTRODUÇÃO}

A industrialização de muitas regiões aumentou a geração de efluentes contendo metais pesados, como o chumbo, que é um dos mais tóxicos. A maioria dos metais pesados é prejudicial a uma variedade de espécies vivas, incluindo os seres humanos. Esses elementos quando descartados como resíduos industriais, na água, no solo ou no ar, podem ser adsorvidos por vegetais e animais, provocando graves intoxicações ao longo da cadeia alimentar e, por não possuir nenhuma função dentro dos organismos, sua acumulação pode provocar sérias doenças, principalmente nos seres humanos, afetando rins e ossos, entre outros.

Métodos convencionais de tratamento de efluentes contendo metais pesados como precipitação, oxidação e/ou redução, filtração, troca iônica, tratamento eletroquímico, dentre outros, são muitas vezes restritos por inviabilidade técnica e/ou econômica, especialmente quando os metais estão presentes em concentrações relativamente baixas. Por exemplo, processos de precipitação nem sempre garantem que a concentração metálica esteja dentro dos níveis máximos estabelecidos pela legislação específica, além de produzir, em alguns casos, resíduos difíceis de serem tratados. Por outro lado, resinas poliméricas (de troca-iônica ou quelantes) são bastante eficientes, porém de alto custo (Bhatnagar et. al, 2013; Gurgel et al., 2008; Gupta, 2004 e Assis, 2012).

Diferentes tipos de biomassa, como produtos agrícolas, madeira, têm a capacidade de reter íons metálicos através de adsorção, levando vantagem sobre resinas comerciais, por serem viáveis economicamente, biodegradáveis e provirem de recursos renováveis (Vaughan et al., 2001). Esta capacidade pode ser aumentada significativamente quando a biomassa é modificada quimicamente. Marshall et al. (1999) demonstraram isto quando submeteram casca de soja a uma extração alcalina $(\mathrm{NaOH})$, seguida de uma modificação com ácido cítrico em temperatura elevada e observaram o aumento da capacidade da biomassa em reter cádmio e cobre. Vaughan et al. (2001) aplicaram os mesmos tratamentos químicos em sabugo de milho e demonstraram que, dependendo do metal ( $\mathrm{Cd}, \mathrm{Cu}, \mathrm{Zn}$ e $\mathrm{Ni}$ ) a ser adsorvido e do tratamento utilizado, a capacidade de carregamento do material é equivalente ou melhor do que o carregamento de determinadas resinas comerciais.

A adsorção de íons metálicos em biomassa vem se destacando e cada vez mais é uma alternativa aos métodos físico-químicos (Bai e Abraham, 2001). O crescimento da utilização da técnica gera a necessidade de estudos que objetivam no aperfeiçoamento do adsorvente. $A$ modificação química do bagaço proveniente da cana-de-açúcar, para o aumento do seu desempenho como adsorvente, tem sido amplamente pesquisado (Karnitz et al., 2007; Gurgel et al., 2008; Assis, 2012).

Devido a estas considerações e a procura por novos adsorventes que sejam viáveis do ponto de vista técnico/econômico este trabalho teve como objetivo avaliar a capacidade do bagaço de cana-de-açúcar, modificado quimicamente, para a retenção do metal chumbo presente em soluções aquosas. 


\section{REVISÃO BIBLIOGRÁFICA}

\subsection{Contaminação Ambiental}

A presença de metais nos ciclos biogeoquímicos no meio ambiente tem ocorrência natural, porém, com o aumento da ação antrópica, especialmente a intensificação da industrialização, tem promovido um aumento significativo da concentração destas substâncias (Assis, 2012).

Os metais pesados apresentam elevada toxicidade, sendo considerados prejudiciais à saúde humana, o que tem motivado a intensa fiscalização quanto ao cumprimento da legislação ambiental.

Consideram-se elementos químicos metálicos como sendo metais de transição e alguns metais representativos que possuem peso especifico acima de 6,0 g/cm ${ }^{3}$ (Manaham, 2001; Moreira, 2008). Em níveis de concentração adequados alguns metais são fundamentais para o bom funcionamento do organismo, como o ferro (Fe), necessário para a produção de hemoglobina (Williams et al., 2000; Karnitz et al., 2007).

O Conselho Nacional do Meio Ambiente, CONAMA, órgão normativo e fiscalizador, através da resolução no 397, de 3 de abril de 2008 apresenta os padrões de lançamentos de efluentes, descrevendo a quantidade máxima de diversas substâncias que podem ser descartadas no meio ambiente (CONAMA, 2008). Algumas destas substâncias são apresentadas na Tabela 1.

Tabela 1: Concentrações máximas permitidas para o descarte de metais em efluentes aquosos.

\begin{tabular}{l|c}
\hline \multicolumn{1}{c|}{ Metal } & Concentração permitida (mg/L) \\
\hline Chumbo $(\mathrm{Pb})$ & 0,5 \\
Cádmio $(\mathrm{Cd})$ & 0,2 \\
Cobre $(\mathrm{Cu})$ & 1,0 \\
Mercúrio $(\mathrm{Hg})$ & 0,01 \\
Cromo hexavalente $\left(\mathrm{Cr}^{6+}\right)$ & 0,1 \\
\hline
\end{tabular}

Fonte: Adaptado da Resolução no 397 do CONAMA, 2008.

A Tabela 2 mostra a aplicação industrial de alguns metais e os efeitos relacionados à exposição dos mesmos para o organismo. 
Tabela 2: Aplicação industrial e efeitos da exposição a metais pesados.

\begin{tabular}{|c|c|c|}
\hline Metal & Aplicações & Efeitos \\
\hline Chumbo (Pb) & $\begin{array}{l}\text { Componente de soldas, lâminas de } \\
\text { proteção contra raios } \mathrm{X} \text { e revestimento } \\
\text { de automóveis. }\end{array}$ & $\begin{array}{l}\text { Danos ao sistema nervoso } \\
\text { central, fraqueza, irritabilidade, } \\
\text { astenia, náusea, anemia e } \\
\text { câncer renal. }\end{array}$ \\
\hline Cádmio (Cd) & $\begin{array}{l}\text { Pigmento para plásticos, pilhas e } \\
\text { baterias ( } \mathrm{Ni}-\mathrm{Cd}) \text { e componentes } \\
\text { eletrônicos. }\end{array}$ & $\begin{array}{l}\text { Náusea, vômito, diarreia, dores } \\
\text { abdominais, fragilidade nos } \\
\text { ossos, câncer pulmonar. }\end{array}$ \\
\hline $\begin{array}{l}\text { Mercúrio } \\
\qquad(\mathrm{Hg})\end{array}$ & $\begin{array}{l}\text { Termômetros, amálgama odontológico, } \\
\text { lâmpada fluorescente e mineração. }\end{array}$ & $\begin{array}{l}\text { Danos ao sistema nervoso, } \\
\text { redução da coordenação } \\
\text { motora, diminuição do campo } \\
\text { visual, perdas de memória e } \\
\text { câncer. }\end{array}$ \\
\hline
\end{tabular}

Fonte: Rocha, A. F. (2009); Sousa, J. M. D., (2010).

\subsection{Chumbo}

O chumbo $(\mathrm{Pb})$ é um elemento não essencial que se acumula no organismo. Como esse metal traz danos à quase todos os órgãos e sistemas do organismo, os mecanismos de toxicidade propostos envolvem processos bioquímicos fundamentais, que incluem a habilidade do chumbo de inibir ou imitar a ação do cálcio e de interagir com proteínas (Moreira e Moreira, 2004).

Os malefícios relacionados à exposição ao chumbo ocorrem especialmente devido a sua interferência no funcionamento das membranas celulares e enzimas, formando complexos estáveis com ligantes contendo enxofre, fósforo, nitrogênio ou oxigênio (grupamentos - $\mathrm{SH},-$ $\mathrm{H}_{2} \mathrm{PO}_{3},-\mathrm{NH}_{2},-\mathrm{OH}$ ), que funcionam como doadores de elétrons.

Os maiores níveis de chumbo são encontrados nos ossos, entretanto, os primeiros efeitos da exposição ao metal não são aí observados; inclusive, sendo ainda pequeno o conhecimento existente sobre os efeitos e mecanismos de ação do chumbo nos ossos (Moreira e Moreira, 2004).

O quadro a seguir contém alguns órgãos e sistemas que podem ser comprometidos pela presença do chumbo e os efeitos da exposição.

\begin{tabular}{|c|c|}
\hline Sistemas e/ou órgãos & Danos \\
\hline Efeitos neurológicos & $\begin{array}{l}\text { Danos ao sistema nervoso central, menor quociente de inteligência, } \\
\text { deficiência cognitiva, dificuldade motora. }\end{array}$ \\
\hline Efeitos hematológicos & Anemia através da inibição da síntese da hemoglobina. \\
\hline Efeitos endocrinológicos & Redução da produção de vitamina D. \\
\hline 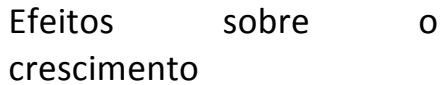 & Redução de peso e circunferência do tórax e crânio. \\
\hline Efeitos renais & $\begin{array}{l}\text { Redução da função renal acompanhada de hipertensão, esclerose vascular, } \\
\text { atrofia ou hiperplasia da célula tubular e fibrose intersticial progressiva }\end{array}$ \\
\hline $\begin{array}{l}\text { Efeitos sobre a reprodução } \\
\text { e desenvolvimento }\end{array}$ & $\begin{array}{l}\text { Maior frequência de abortos e partos de natimortos e indícios da redução } \\
\text { da produção de esperma. }\end{array}$ \\
\hline
\end{tabular}




\begin{tabular}{|l|l|}
\hline Efeitos carcinogênicos & Potencial causador de câncer renal \\
Efeitos cardiovasculares & Hipertensão \\
Efeitos gastrintestinais & Cólica \\
\hline
\end{tabular}

Fonte: Adaptado de Moreira e Moreira, 2004.

\subsection{Bagaço de cana-de-açúcar}

Atualmente o Brasil é o país com maior área plantada de cana-de-açúcar no mundo. De acordo com a Companhia Nacional de Abastecimento, CONAB, é esperado que o plantio para a safra 2017/2018 atinja nove milhões de hectares (CONAB, 2018).

Ainda sobre a safra 2017/2018 a Tabela 3 apresenta uma estimativa de produção de açúcar e etanol para cada região do Brasil.

Tabela 3: Produção de cana-de-açúcar por região na safra 2017/2018.

\begin{tabular}{l|c|c}
\hline \multicolumn{1}{c|}{ Região } & Açúcar (1.000 t) & Etanol total (1.000 L) \\
\hline Norte & 48,5 & $232.445,0$ \\
Nordeste & $3.514,0$ & $1.906 .908,4$ \\
Centro-Oeste & $3.755,4$ & $7.755 .161,0$ \\
Sudeste & $25.318,9$ & $17.144 .826,9$ \\
Sul & $2.923,3$ & $1.620 .582,5$ \\
Total & $35.560,2$ & $28.659 .923,7$ \\
\hline
\end{tabular}

Fonte: CONAB abril/2018.

A produção de cana para a safra $2017 / 2018$ foi de 633,36 milhões de toneladas. Estima-se que $43,1 \%$ da cana-de-açúcar seja moída para a produção de açúcar, gerando aproximadamente 37,87 milhões de toneladas de açúcar. Os 56,9\% restantes da matéria prima destinados a produção de etanol tem capacidade de produzir 28,65 bilhões de litros deste álcool (CONAB, 2018).

Estima-se que a quantidade de bagaço gerado por tonelada de cana-de-açúcar é 292,43 $\mathrm{kg} / \mathrm{t}$, logo, de acordo com a previsão da colheita na safra $2017 / 2018$ espera-se que seja gerado 185,6 milhões de toneladas de bagaço de cana-de-açúcar (CONAB, 2018).

O bagaço da cana-de-açúcar é um resíduo agrícola gerado em grande quantidade e rica em biomassa lignínica celulósica renovável (Karnitz et al., 2007), o bagaço representa aproximadamente $30 \%$ da massa da cana (Silva et al., 2007; Pereira, 2008).

Um grande número de usinas tem aproveitado este resíduo como combustível para a geração de vapor nas caldeiras, utilizado para impulsionar as turbinas de geração de energia elétrica. $O$ bagaço excedente é comercializado para empresas que processam esse material.

Estudos têm mostrado que a utilização de biomassa como adsorvente para a remoção de metais pesados e corantes de efluentes industriais é viável (Dallago e Smaniotto, 2005; Vadivelan e Kumar 2005; Ofomaja, 2008), abrindo mais um espaço no mercado para as empresas especializadas no processamento destes materiais, em especial o bagaço da cana-de-açúcar. 
A composição química do bagaço da cana-de-açúcar varia de acordo com o tipo da cana, o manuseio, o tipo de solo e a forma de colheita. A Tabela 4 apresenta as composições química, físico-química e da fibra do bagaço.

Tabela 4: Composição média do bagaço de cana-de-açúcar.

\begin{tabular}{l|c}
\multicolumn{1}{c|}{ Composição química média } & (\%) \\
\hline Carbono & $39,7-49$ \\
Oxigênio & $40-46$ \\
Hidrogênio & $5,5-7,4$ \\
Nitrogênio e Cinzas & $0-0,3$ \\
\hline \multicolumn{1}{c}{ Propriedades físico-químicas } & $\mathbf{( \% )}$ \\
\hline Umidade & 50 \\
Fibra & 46 \\
Brix & 2 \\
Impurezas minerais & 2 \\
\hline \multicolumn{1}{c}{ fibra do bagaço da cana-de-açúcar } & $\mathbf{( \% )}$ \\
\hline Celulose & $26,6-54,3$ \\
Hemicelulose & $14,3-24,4$ \\
Lignina & $22,7-29,7$ \\
\hline
\end{tabular}

Fonte: Adaptado de Silva et al., 2007

A hidrólise ácida do bagaço da cana-de-açúcar promove a protonação do oxigênio da ligação B-glucosídica, em seguida a ligação se rompe deixando o carbono com carga positiva, polarizando ainda mais a molécula, os grupos hidroxílicos da celulose são nucleófilos e doam pares de elétrons para o chumbo estabelecendo assim a relação de adsorção (Vicente, 2011). A Figura 1 apresenta a hidrólise ácida da celulose.

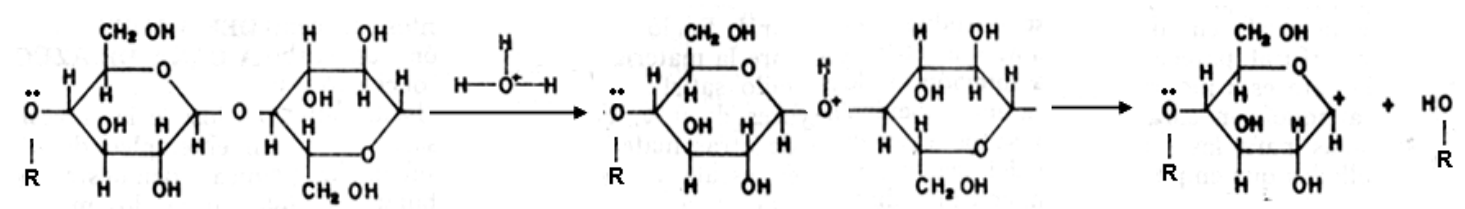

Figura 1: Hidrólise ácida da celulose.

\subsection{Adsorção}

A adsorção é um processo de transferência de um ou mais constituintes de uma fase fluida para a superfície de uma fase sólida. Atualmente, a adsorção é definida como um fenômeno de superfície no qual uma concentração finita de moléculas de um fluido, por afinidade, adere a uma superfície devido a um não balanceamento de forças. Os principais elementos da adsorção são o fluido (adsortivo), a superfície na qual o fenômeno ocorre (adsorvente) e os componentes retidos pela superfície (adsorbato). Os dois tipos principais de adsorção são a física e a química (Ruthven, 1984).

A adsorção física é um processo reversível no qual uma molécula entra em contato com a superfície do meio filtrante e adere a ela através de forças mecânicas. A energia, por exemplo a 
cessão de calor, pode causar às moléculas o desligamento da superfície do meio filtrante. Dessa maneira, o meio filtrante pode ser regenerado e reusado.

$\mathrm{Na}$ adsorção física, os efeitos atrativos que ocorrem entre o adsorvente e o adsorbato são relativamente fracos, envolvendo, principalmente, interações eletrostáticas e de Van der Waals, o que compreende as interações de polarização, tipo dipolos permanente, dipolos induzido e quadrupolo. As contribuições de Van der Waals estão sempre presentes, enquanto as contribuições eletrostáticas são significantes apenas no caso de adsorventes que possuem uma estrutura iônica, tais como zeólitas (Ruthven, 1984).

A energia liberada quando uma espécie é adsorvida fisicamente é da mesma ordem de grandeza da entalpia de condensação de moléculas (1 a $5 \mathrm{~kJ} \cdot \mathrm{mol}^{-1}$ ) (Ruthven, 1984). A pequena variação da entalpia é insuficiente para resultar em uma quebra de ligação, portanto uma espécie fisissorvida preserva sua identidade química, sendo assim, esse tipo de adsorção tem caráter não específico. Essa propriedade indica que o mesmo fenômeno pode ser observado para diferentes adsorbatos, sendo necessário que apresente apenas dipolo induzido (Ruthven, 1984). A adsorção física é do tipo não localizada, ou seja, as moléculas adsorvidas podem se movimentar livremente sobre a superfície do adsorvente.

No caso da adsorção química, ou quimissorção, tem-se um compartilhamento ou uma troca de elétrons entre o adsorvente e o adsorbato. Esse fenômeno faz com que a adsorção perca suas características puramente físicas, passando a haver ligações covalentes ou iônicas entre os constituintes do processo adsortivo. Pode-se dizer que as energias envolvidas durante o processo de quimissorção se aproximam de valores observados em reações químicas.

O processo de adsorção pode ser influenciado por vários fatores, tais como: temperatura, natureza e concentração do adsorbato, natureza do adsorvente e seu estado de agregação, pH, tamanho de partícula, tempo de contato e velocidade de agitação (Curbelo, 2002).

Experimentalmente, um sistema adsorbato-adsorvente é considerado em equilíbrio quando, por maior que seja o tempo de contato entre o adsorvente e a espécie a ser adsorvida, não há variação na concentração da fase fluida. Este equilíbrio de fases pode ser representado por isotermas de adsorção, ou seja, curvas a temperatura constante que relacionam os dados entre a concentração do adsorbato e a massa do adsorvente.

Um dos modelos adsortivos mais estudados é o proposto por Langmuir (1918). Este considera a formação de uma única camada sobre o adsorvente, podendo também existir a formação de multicamadas através dos mecanismos de evaporação e condensação. A isoterma de Langmuir corresponde a um tipo altamente idealizado na qual são feitas as seguintes hipóteses:

- as moléculas são adsorvidas em pontos discretos da superfície que são chamados "sítios de adsorção";

- cada sítio pode acomodar somente uma entidade adsorvida (adsorção em monocamada).

- a energia da espécie adsorvida é a mesma em qualquer ponto da superfície e é independente da presença ou ausência de moléculas adsorvidas na vizinhança, ou seja, a superfície é completamente uniforme sob o ponto de vista energético.

- a adsorção é localizada e ocorre por colisão de moléculas com sítios disponíveis.

A Equação 1 é comumente denominada de Equação de Langmuir e é utilizada nos casos no qual se considera o equilíbrio de adsorção e dessorção física em monocamada: 


$$
q_{e}=\frac{K_{L} C_{e} q_{m}}{1+K_{L} C_{e}}
$$

Em que $q_{e}(\mathrm{mg} / \mathrm{g})$ corresponde à massa do soluto adsorvida por unidade de massa do adsorvente no equilíbrio; $C_{e}(\mathrm{mg} / \mathrm{L})$, a concentração de equilíbrio do adsorbato na fase fluida; $K_{L}$ $(\mathrm{L} / \mathrm{mg})$ é a constante de equilíbrio relacionada com a energia de ligação adsorbato-adsorvente; $q_{m}$ $(\mathrm{mg} / \mathrm{g})$ é constante que representa a quantidade máxima de adsorbato que pode ser adsorvida. A determinação das constantes $\mathrm{K}_{\mathrm{L}}$ e $\mathrm{q}_{\mathrm{m}}$ podem ser realizadas a partir de uma regressão não-linear, de modo a adequar o modelo aos valores experimentais.

Dentre os modelos de adsorção clássicos, a equação de Freundlich foi uma das primeiras equações propostas para estabelecer uma relação entre a quantidade de material adsorvido e a concentração do material na solução (Araújo e Sobrinho, 2000). Este modelo não considera a equivalência energética dos sítios de adsorção, admitindo que o processo de adsorção apresenta uma distribuição exponencial de calores de adsorção a partir da monocamada adsorvida e pode assim ser representado:

$q_{e}=K_{F} C_{e}^{1 / n}$

Em que qe $(\mathrm{mg} / \mathrm{g})$ e Ce $(\mathrm{mg} / \mathrm{L})$ têm o mesmo significado daqueles já definido para a isoterma de Langmuir, $\mathrm{KF}(\mathrm{L} / \mathrm{g})$ e $\mathrm{n}$ (adimensional) são respectivamente constantes empíricas indicadoras da capacidade e intensidade da energia de adsorção (Levan et al., 2007) Do mesmo modo, pode-se determinar os parâmetros $n$ e KF a partir de uma regressão não-linear. $O$ valor de $n$ é sempre maior que 1; quando se encontra no intervalo entre 1 e 10 é um indicativo da natureza favorável da adsorção (Erdem et al., 2004)

\section{METODOLOGIA}

\subsection{Ativação química do bagaço}

Nesta etapa foi realizada a lavagem do bagaço da cana para a remoção de resíduos sólidos que pudessem causar problemas nas etapas seguintes do processo como areia, palha ou madeira.

Após a etapa de lavagem o bagaço foi seco em estufa à $60{ }^{\circ} \mathrm{C}$ por 40 horas, em seguida foi triturado em um moinho de facas e peneirado utilizando peneiras de série de Tyler para obter partículas com diâmetro entre 0,5 e 2,0 mm.

Por fim, o bagaço da cana-de-açúcar foi submetido (estaticamente) a um tratamento químico com ácido sulfúrico $\left(1,0 \mathrm{molL}^{-1}\right)$ com a finalidade de modificá-lo e ativar suas propriedades como adsorvente. Nesse processo o bagaço foi submetido a solução de ácido sulfúrico por 24 horas, a relação sólido-líquido utilizada nesta etapa foi de $0,05 \mathrm{~g}$ de bagaço por $\mathrm{mL}$ de ácido. Em seguida, foi realizada a filtração do bagaço para a remoção do ácido, e logo após o bagaço foi lavado com água destilada até o pH estabilizar e ficar próximo a 6 . 


\subsection{Preparação das soluções de chumbo}

Para realizar o estudo de adsorção, foram utilizadas soluções aquosas (sintéticas) de chumbo. Seis soluções foram preparadas a partir do sal nitrato de chumbo, $\mathrm{Pb}(\mathrm{NO} 3) 2$, com concentrações do cátion de 2, 5, 10, 20, 40, 60 e 80 ppm.

\subsection{Determinação da concentração do chumbo}

A determinação da concentração de chumbo remanescente em meio aquoso será realizada a partir do método de espectrofotometria no UV.

Em um espectrofotômetro UV (marca Shimadzu), foi construída uma curva de calibração para a determinação de chumbo $\left(\mathrm{Pb}^{2+}\right)$ no comprimento de onda de $408 \mathrm{~nm}$. As soluções utilizadas nesta etapa foram preparadas conforme item 3.2.

A concentração de chumbo presente na fase fluida após atingir o equilíbrio é calculada com base na leitura da absorbância, que com o auxílio da equação da reta da curva de calibração, é possível calcular a concentração do chumbo.

\subsection{Isotermas de adsorção}

A obtenção das isotermas de adsorção se basearam no método do banho finito. Massas conhecidas e iguais a 0,5 g de bagaço de cana-de-açúcar, ativado quimicamente, foram postas em contato com $50 \mathrm{~mL}$ de soluções aquosas de chumbo com diferentes concentrações $(2,5,10,20$, 40, 60 e 80 ppm). Os experimentos foram realizados nas temperaturas de 30,45 e $60{ }^{\circ} \mathrm{C}$. $\mathrm{O}$ equipamento utilizado foi o Banho Metabólico Dubnof.

Após 24 horas de contato entre as fases, tempo considerado suficiente para o sistema atingir o equilíbrio, a solução remanescente foi analisada e a concentração de chumbo determinada. Com a obtenção da concentração final de equilíbrio do chumbo na fase aquosa pode-se calcular o parâmetro $q_{\text {ExP }}$ (Equação 3) que expressa a quantidade de chumbo adsorvida por massa de adsorvente. Este parâmetro é comumente expresso em $\mathrm{mg}$ de adsorbato/g de adsorvente:

$q_{E X P}=\frac{V\left(C_{i}-C_{e}\right)}{m}$

Em que $V(L)$ é o volume da solução, $C_{i}(\mathrm{mg} / \mathrm{L})$ e $C_{e}(\mathrm{mg} / \mathrm{L})$ são, respectivamente, as concentrações inicial e final de equilíbrio do chumbo no meio aquoso, $\mathrm{m}$ ( $\mathrm{g}$ ) é a massa de adsorvente e $q_{\operatorname{Exp}}(\mathrm{mg} / \mathrm{g})$ representa a massa de chumbo adsorvido pela massa de adsorvente. Um gráfico de $q_{\text {Exp }}$ versus $C_{e}(\mathrm{mg} / \mathrm{L})$ é plotado e, assim, tem-se a isoterma de adsorção. Com isso, os modelos de Langmuir e Freundlich foram ajustados aos dados experimentais. 
Para o ajuste dos modelos, assim como na determinação dos parâmetros característicos das Equações 1 e 2 para Langmuir e Freundlich, respectivamente, foi utilizado a técnica da regressão não-linear com o auxílio do software Estatística 7.0, de modo a verificar a consistência dos dados com um nível de confiança de $95 \%(\alpha=0,05)$.

\subsection{Parâmetros termodinâmicos}

A variação da energia livre de Gibbs $(\Delta G)$, a variação de entalpia $(\Delta H)$ e a variação de entropia $(\Delta S)$ podem ser calculadas a partir de dados obtidos das isotermas de adsorção, o conhecimento destes parâmetros podem oferecer informações relevantes como a espontaneidade do processo, o caráter endotérmico ou exotérmico do experimento e o tipo de adsorção realizada (Schneider, 2008).

Com a constante de adsorção $K_{\text {ads }}$ pode-se, através da Equação 4 apresentada abaixo, obter

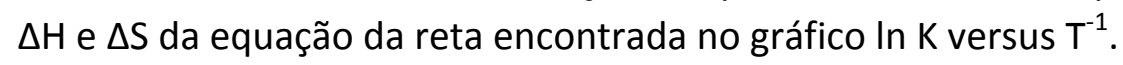

$\ln K_{a d s}=\frac{\Delta S}{R}-\frac{\Delta H}{R T}$

A energia livre de Gibbs pode ser determinada através das Equações 4 e 5.

$\Delta G=-R T \ln K_{a d s}$

$\Delta S=\frac{\Delta H-\Delta G}{T}$

Em que: T é a temperatura em Kelvin (K); R a constante universal dos gases $\left(\mathrm{Jmol}^{-1} \mathrm{~K}^{-1}\right) ; \Delta \mathrm{G}$ a variação da energia livre de Gibbs $\left(\mathrm{Jmol}^{-1}\right) ; \Delta \mathrm{S}$ a variação da entropia $\left(\mathrm{Jmol}^{-1} \mathrm{~K}^{-1}\right) ; \Delta \mathrm{H}$ a variação da entalpia $\left(\mathrm{Jmol}^{-1}\right)$ e $\mathrm{K}_{\mathrm{ads}}(\mathrm{L} / \mathrm{mg})$ é a constante de equilíbrio relacionada com a energia de ligação adsorbato-adsorvente.

\section{RESULTADOS E DISCUSSÃO}

\subsection{Curva de calibração}

Para registrar a concentração de equilíbrio na fase fluida após o ensaio de adsorção, foi construída a curva de calibração de $\mathrm{Pb}^{2+}$ em um espectrofotômetro UV utilizando soluções de diferentes concentrações do cátion como apresentado na Figura 2. 


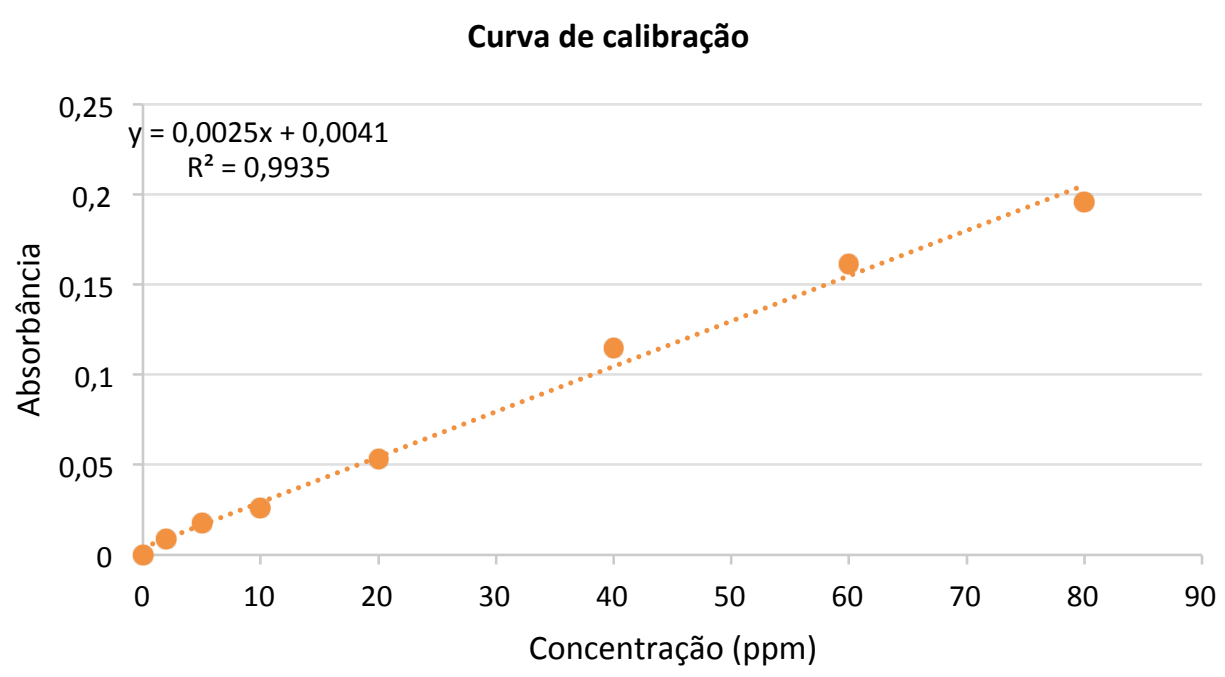

Figura 2: Curva de calibração de chumbo.

\subsection{Isotermas de adsorção}

A Figura 3 apresenta as isotermas de adsorção de $\mathrm{Pb}^{2+}$ nas diferentes temperaturas estudadas. Da análise desta figura, observa-se que adsorção é mais favorecida à temperatura de $30{ }^{\circ} \mathrm{C}$, o que remete ao caráter exotérmico desta técnica de separação. De acordo com Gusmão (2011) os dados experimentais se enquadram como isoterma favorável. A adsorção máxima ( $q_{m}$ ) obtida para a temperatura de $30{ }^{\circ} \mathrm{C}$ é de aproximadamente $4,7 \mathrm{mg}$ de chumbo/g de bagaço.

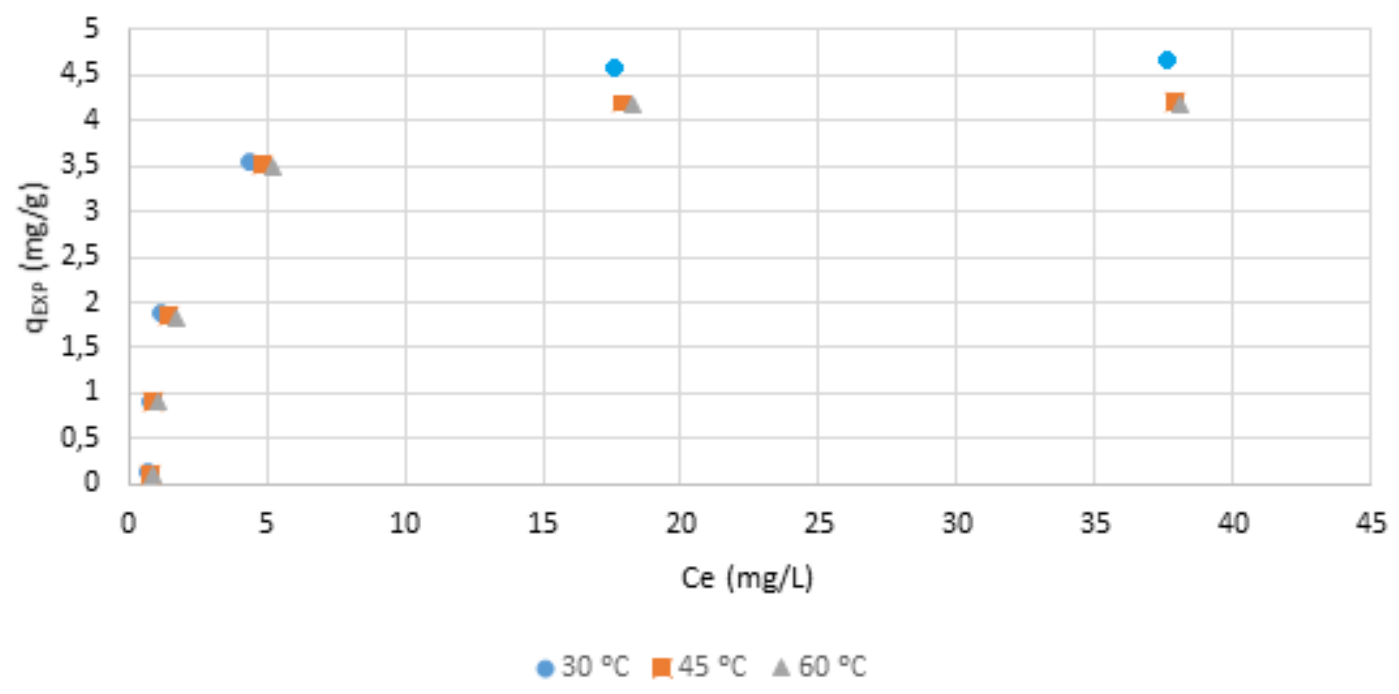

Figura 3: Isotermas de adsorção.

Na Tabela 5 para o modelo de Langmuir, tem-se os seguintes valores para os parâmetros $\mathrm{K}_{\mathrm{L}}$ e $q_{m}$, que são, respectivamente, a constante de equilíbrio relacionada com a energia de ligação 
adsorbato-adsorvente e a constante que representa a quantidade máxima de adsorbato que pode ser adsorvida:

Tabela 5: Valores dos parâmetros $\mathrm{K}_{\mathrm{L}}$ e $\mathrm{q}_{\mathrm{m}}$ obtidos a partir da regressão não-linear.

\begin{tabular}{c|c|c|c}
\hline Temperatura $\left({ }^{\circ} \mathbf{C}\right)$ & $\mathbf{K}_{\mathbf{L}}(\mathbf{L} / \mathbf{m g})$ & $\mathbf{q}_{\mathbf{m}}(\mathbf{m g} / \mathbf{g})$ & $\mathbf{R}^{\mathbf{2}}$ \\
\hline 30 & 0,38 & 4,87 & 0,967 \\
45 & 0,33 & 4,31 & 0,952 \\
60 & 0,29 & 4,12 & 0,938 \\
\hline
\end{tabular}

Na Tabela 6 para o modelo de Freundlich, tem-se os seguintes valores para os parâmetros $\mathrm{K}_{\mathrm{F}}$ e $\mathrm{n}$, que são, respectivamente, as constantes empíricas indicadoras da capacidade e da intensidade da energia de adsorção:

Tabela 6: Valores dos parâmetros $\mathrm{K}_{\mathrm{F}}$ e $\mathrm{n}$ obtidos a partir da regressão não-linear.

\begin{tabular}{c|c|c|c}
\hline Temperatura $\left({ }^{\circ} \mathbf{C}\right)$ & $\mathbf{K}_{\mathbf{F}}(\mathbf{L} / \mathbf{g})$ & $\mathbf{n}$ & $\mathbf{R}^{\mathbf{2}}$ \\
\hline 30 & 1,49 & 3,03 & 0,829 \\
45 & 1,41 & 2,94 & 0,832 \\
60 & 1,33 & 2,86 & 0,833 \\
\hline
\end{tabular}

A análise das Tabelas 5 e 6 mostra que o processo adsortivo não segue o modelo de Freundlich, pois o ajuste $\left(R^{2}\right)$ dos dados experimentais foi baixo, indicando que as constantes $K_{L} e$ n não são estatisticamente significativas. Já o modelo de Langmuir apresentou um bom resultado, isto é, o processo adsortivo pode ser descrito por este modelo e as constantes determinadas $\mathrm{q}_{\mathrm{m}} \mathrm{e}$ $\mathrm{K}_{\mathrm{L}}$ apresentam significância estatística.

Uma outra análise confirma o exposto anteriormente. Os valores-p para as constantes do modelo de Langmuir $q_{m}$ e $K_{L}$ foram 0,0132 e 0,0121, respectivamente, ou seja, menores do que o nível de significância adotado $(\alpha=0,05)$. Este fato confirma a significância estatísticas destes parâmetros. Resultado diferente foi encontrado para as constantes $K_{F}$ e $n$ do modelo de Freundlich, onde os valores-p encontrados foram 0,8212 e 0,8431, respectivamente, sendo estes superiores ao nível de significância adotado, o que consequentemente torna estes parâmetros sem significância estatística.

A Figura 4 mostra o ajuste dos dados experimentais ao modelo de Langmuir para temperatura de $30{ }^{\circ} \mathrm{C}$, que apresentou os maiores valores de adsorção de chumbo. 


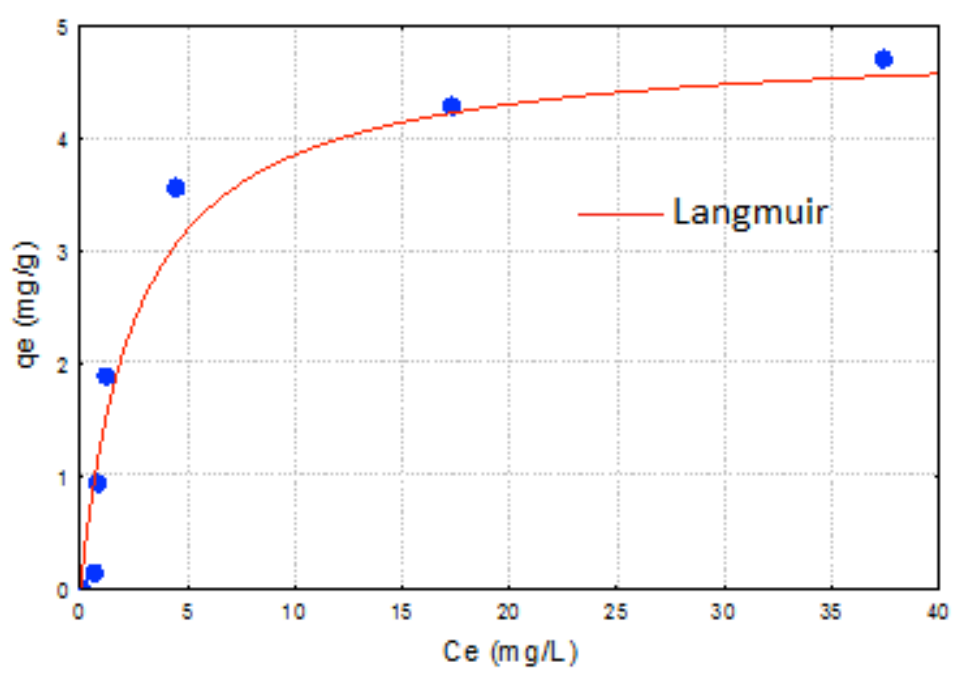

Figura 4: Ajuste dos dados experimentais ao modelo de Langmuir.

Analisando a Figura 4 confirma-se o bom ajuste dos dados experimentais ao modelo de Langmuir e ao mesmo tempo verifica-se o valor da adsorção máxima $\left(q_{m}\right)$ que é de aproximadamente $4,7 \mathrm{mg} / \mathrm{g}$, valor este confirmado pelos dados Figura 3 e da Tabela 5 .

\subsection{Parâmetros Termodinâmicos}

Os parâmetros termodinâmicos, apresentados na Tabela 7, foram calculados com base na isoterma de Langmuir. A partir da equação de Van't Hoff (Equação 4) obtém-se a equação da reta (Figura 5) que permite calcular a variação de entalpia $(\Delta H)$ e a variação de entropia $(\Delta S)$. Utilizando a Equação 4 calculou-se a energia livre de Gibbs $(\Delta G)$.

\section{Parâmetros Termodinâmicos}

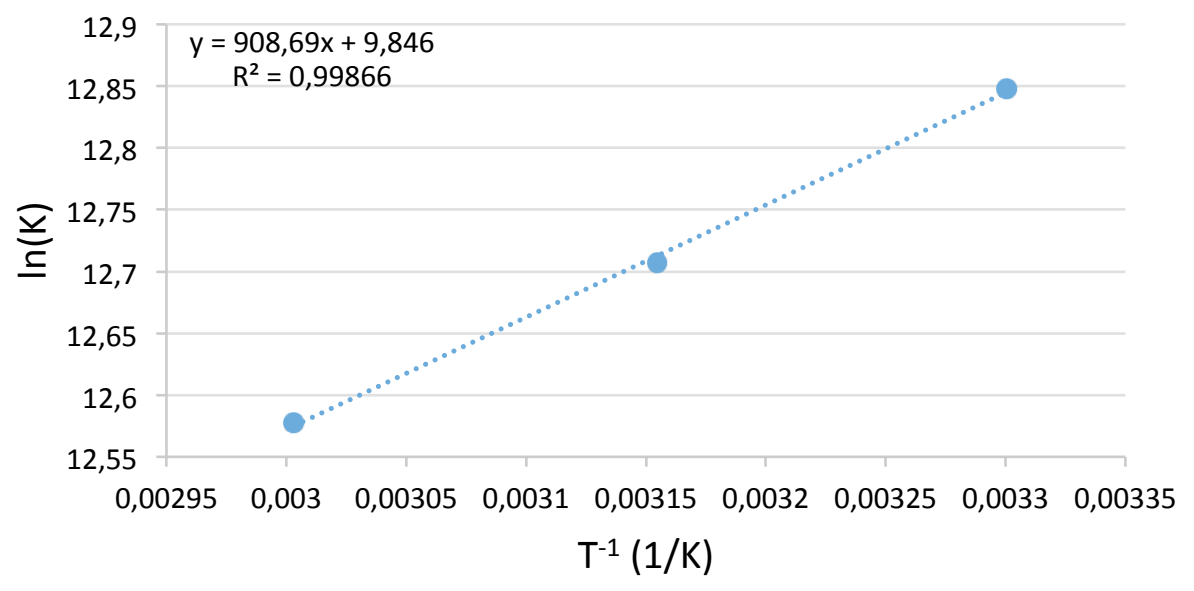

Figura 5: Gráfico de Van't Hoff para a adsorção de chumbo em bagaço modificado. 
Tabela 7: Parâmetros termodinâmicos.

\begin{tabular}{c|c|c|c}
\hline Temperatura $\left({ }^{\mathbf{0}} \mathbf{C}\right)$ & $\Delta \mathbf{G}\left(\mathrm{KJmol}^{-1}\right)$ & $\Delta \mathbf{H}\left(\mathrm{KJmol}^{-1}\right)$ & $\Delta \mathbf{S}\left(\mathrm{KJmol}^{-1} \mathrm{~K}^{-1}\right)$ \\
\hline 30 & $-32,37$ & & \\
45 & $-33,59$ & $-7,55$ & 0,082 \\
60 & $-33,78$ & & \\
\hline
\end{tabular}

Os parâmetros termodinâmicos (Tabela 7) indicam que o processo é exotérmico e situa-se próximo a zona que caracteriza uma adsorção física, pois o $\Delta \mathrm{H}$ tem ordem de grandeza de 1 a 5 $\mathrm{KJmol}^{-1}$ como foi descrito por Ruthven (1984), o processo ocorre de forma espontânea, segundo a energia livre de Gibbs, deixando ainda mais claro que é viável a utilização deste adsorvente para a remoção de chumbo de efluentes industriais. Além disso, o $\Delta G$ também aponta para uma fisissorção de acordo com Liu (2010) pois o valor de energia livre de Gibbs para esse tipo de adsorção situa-se entre $-20 \mathrm{kJmol}^{-1}$ e $0 \mathrm{kJmol}^{-1}$, próximos aos valores encontrados neste trabalho, enquanto que para a adsorção química o valor varia entre $-400 \mathrm{kJmol}^{-1}$ e -80 valor $\mathrm{kJmol}^{-1}$.

\section{CONCLUSÕES}

O bagaço de cana-de-açúcar, quimicamente modificado, apresentou-se como uma boa alternativa para a remoção de chumbo de soluções aquosas, destacando-se a adsorção à $30{ }^{\circ} \mathrm{C}$ por não necessitar do aumento de temperatura e apresentar os valores maiores de adsorção do metal.

Os dados experimentais de equilíbrio (isotermas de adsorção) para o sistema solução aquosa de chumbo-bagaço de cana-de-açúcar ativado foram bem representados pelo modelo de adsorção de Langmuir.

Os parâmetros termodinâmicos determinados comprovam o caráter exotérmico do processo de adsorção e a natureza física (adsorção física) do processo estudado".

\section{REFERÊNCIAS}

Araújo, W., \& Sobrinho, N. A. (2000). Influência das propriedades físicas e químicas de solos intemperizados na adsorção de chumbo, cobre e zinco. Floresta e Ambiente, 7(1), 167-180.

Assis, P. A. (2012). Adsorção de metais pesados em solução aquosa e drenagem ácida de mina utilizando bagaço de cana-de-açúcar modificado quimicamente. Dissertação de mestrado. Pós-Graduação em Engenharia Ambiental, Ouro Preto, MG, UFOP.

Bai, S. \& Abraham, T. E. (2001). Biosorpiton of Cr (VI) from aqueous solution by Rhizopus nigricans. Bioresource Technology, v. 79, n. 1, p. 73-81,.

Bhatnagar, A., Hogland, W., Marques, M., \& Sillanpää, M. (2013). An overview of the modification methods of activated carbon for its water treatment applications. Chemical Engineering 
Journal, 219, 499-511. https://doi.org/10.1016/j.cej.2012.12.038

CONAB (2018). Companhia Nacional de Abastecimento. Acompanhamento de safra brasileira: cana-de-açúcar, levantamento em abril de 2018.

CONAMA. (2008). Resolução n 397, 3 de abril de 2008.

Curbelo, F. D. da S. (2002). Estudo da remoção de óleo em águas produzidas na indústria de petróleo, por adsorção em coluna utilizando a vermiculita expandida e hidrofobizada. Dissertação de mestrado. Pós-graduação em Engemharia Química-PPGEQ- UFRN.

Dallago, R. M. \& Smaniotto (2005). A. Resíduos sólidos de curtumes como adsorventes para a remoção de corantes em meio aquoso. Química Nova. Vol. 28, n 3, 433-437.

Erdem, E., Karapinar, N., \& Donat, R. (2004). The removal of heavy metal cations by natural zeolites. Journal of Colloid and Interface Science, 280(2), 309-314. https://doi.org/10.1016/j.jcis.2004.08.028

Gupta, V. \& Ali, I. (2004). Removal of lead and chromium from wastewater using bagasse fly ash a sugar industry waste. Journal of Colloid and Interface Science, p. 321-328, 2004. https://doi.org/ 10.1016/j.jcis.2003.11.007:

Gurgel, L., Freitas, R. P. \& Gil, L. F. (2008). Adsorption of Cu (II), Cd (II), and Pb (II) from aqueous single metal solutions by sugarcane bagasse and mercerized sugarcane gabasse chemically modified with succinic anhydride. Carbohydrate Polymers, p. 922-929, 2008. http://www.repositorio.ufop.br/handle/123456789/5034

Gusmão, K. A. G. (2011). Estudo de adsorção em solução aquosa de dois corantes catiônicos e uma eteramina usando bagaço de cana modificado quimicamente. Dissertação de mestrado. Pós-Graduação em Engenharia Ambiental-PROAMB, Ouro Preto/MG, UFOP.

Karnitz, O., Gurgel, L. V. A., Melo, J. C. P., Botaro, V. R., Melo, T. M. S. \& Gil, R. P. F. (2007) Adsorption of heavy metal ion from aqueous single metal solution by chemically modified sugarcane bagasse. Bioresource Technology, p. 1291-1297, 2007. https://doi.org/ 10.1016/j.biortech.2006.05.013.

Langmuir, I. (1918). The Adsorption of Gases on Plane Surfaces of Glass, Mica and Platinum. Journal of the American Chemical Society, 40 (1914), 1361-1403. https://doi.org/doi: 10.1021/ja02242a004

LeVan, M. D., Carta, G., \& Yon, C. M. (2007). Section 16: Adsorption and Ion Exchange. Perry's Chemical Engineers' Handbook 8/E (8a). McGraw-Hill. https://doi.org/10.1036/0071511393

Manahan, S. E. (2001) Fundamentals of environmetal chemistry, 2aeEd., CRC Press LLC, Boca Raton, $1024 p$.

Marshall, W. E., Wartelle, L. H., Boler, D. E. \& Toles, C. A. (1999). Enhanced metal adsorption by soybean hulls modified with citric acid. Bioresource Technology, v. 69, p. 263-268.

Moreira, F. R. \& Moreira, J. C. (2004). Os efeitos do chumbo sobre o organismo humano e seu significado para a saúde. Revista Panamericana Salud Publica, 15(2) pág 119-129.

Moreira, S. A. (2008). Adsorção de íons metálicos de efluente aquoso usando bagaço do pendúculo de caju: Estudo de batelada e coluna de leito fixo. Dissertação de mestrado. Pós-Graduação em Engenharia Civil, Fortaleza - CE, UFC. 
Ofomaja, A. E. Kinetic study and sorption mechanism of methylene blue and methyl violet onto mansonia (Mansonia altissima) wood sawdust. (2007). Chemical Engineering Journal. Vol. 143 (1-3), p. 85-95. DOI 10.1016/j.cej.2007.12.019

Pereira, F. V. (2008). Remoção de íons zinco (II) de efluentes derivados de processos de galvanoplastia utilizando rejeitos de fibras vegetais modificadas quimicamente. Dissertação de mestrado. Pós-Graduação em Engenharia Ambiental, PROAGUA, Ouro Preto/MG, UFOP.

Rocha, A. F. (2009). Cádmio, Chumbo, Mercúrio - A problemática destes metais pesados na Saúde Pública. Monografia. Universidade do Porto. Faculdade de Ciências da Alimentação e Nutrição. Cidade do Porto-Portugal.

Ruthven, D. (1984). Principles of adsorption and adsorption processes. Chemical Engineering and Processing: Process Intensification (Vol. 19). https://doi.org/10.1016/02552701(85)80013-1

Schneider, L. E. (2008). Adsorção de compostos fenólicos sobre carvão ativado. Dissertação de mestrado. Pós-Graduação em Engenharia Química, Toledo/PR, UNIOESTE.

Silva, V. L. M. M., Gomes, W. C. \& Alsina, O. L. S. (2007). Utilização do bagaço de cana-de-açúcar como biomassa adsorvente na adsorção de poluentes orgânicos. Revista Eletrônica de Materiais e Procesos, vol.1, pág. 27-32.

Sousa, J. M. D., (2010). Exposição a metais pesados no ambiente de trabalho: estabelecimento de bioindicadores de exposição a poluentes. Dissertação de mestrado. Mestrado em Biologia Humana e Ambiente. Universidade de Lisboa, Faculdade de Ciências, Departamento de Biologia animal. Lisboa-Portugal.

Vadidelan, V. \& Kumar, K. V (2005) Equilibrium, kinetics, mechanism, and process design for the sorption of methylene blue onto rice husk. Journal of colloid and Interface Science, V. 286, p. 90-100. https://doi.org/ 10.1016/j.jcis.2005.01.007.

Vaughan, T., Seo W. C. \& Marshall, W. E. (2001). Removal of selected metal ions from aqueous solution using modified corncobs. Bioresource Technology, V. 78, p. 133-139.

Vicente, I. A. (2011). Adsorción de metales pesados de aguas residuales utilizando bagazo de caña de azúcar como adsorbente. Tese de doutorado. Universidad de la Habana/Habana/Cuba.

Williams, P. L., James, R. C. \& Roberts, S. M. (2000). Principles of toxicology: Environmental and industrial applications. 2aEd., John Wiley \& sons, INC, 603p. 